\title{
Tendencias de las búsquedas de información sobre las colecciones SciELO, Redalyc y Dialnet realizadas a través de Google
}

\author{
Cristina Bojo-Canales*, María Sanz-Lorente**, Javier Sanz-Valero*** \\ * Instituto de Salud Carlos III, Biblioteca Nacional de Ciencias de la Salud. Madrid. \\ Correo-e: cbojo@isciii.es | ORCID iD: http://orcid.org//0000-0002-0330-171X \\ ** Universidad Miguel Hernández. Departamento de Salud Pública e Historia de la Ciencia, Campus de Sant Joan d’Alacant. Alicante. \\ Consellería de Sanidad Universal y Salud Pública de la Generalitat Valenciana. Centro de Salud Pública. Manises. \\ Correo-e: msanzlor@gmail.com | ORCID iD: https://orcid.org/0000-0002-8735-0075 \\ *** Universidad Miguel Hernández. Departamento de Salud Pública e Historia de la Ciencia, Campus de Sant Joan d’Alacant. Alicante. \\ Instituto de Salud Carlos III, Escuela Nacional de Medicina del Trabajo. Madrid. España. \\ Correo-e: fj.sanz@isciii.es | ORCID iD: https://orcid.org/0000-0002-8735-0075
}

Recibido: 30-03-20; 2a versión: 19-05-20; Aceptado: 21-05-20; Publicado: 27-04-2021

Cómo citar este artículo/Citation: Bojo-Canales, C.; Sanz-Lorente, M.; Sanz-Valero, J (2021). Tendencias de las búsquedas de información sobre las colecciones SciELO, Redalyc y Dialnet realizadas a través de Google. Revista Española de Documentación Científica, 44 (2), e294. https://doi.org/ 10.3989/redc.2021.2.1765

Resumen: El objetivo del presente estudio fue analizar la tendencia de las búsquedas de información sobre las colecciones bibliográficas SciELO, Redalyc y Dialnet, que se realizan a través de Google. A partir de los resultados obtenidos se observó un claro predomino en las búsquedas, en su país de origen y filiación de cada una de las colecciones: SciELO en Brasil, Redalyc en México y Dialnet en España. La colección más buscada fue SciELO en todo el periodo de tiempo analizado y si bien se constató un crecimiento en el volumen de búsqueda relativo (VBR) tanto en las búsquedas sobre Redalyc y Dialnet, no sucedió así en el de SciELO. En ninguna de ellas se pudo observar un hito claramente destacable. Solo se pudo demostrar una moderada asociación entre los VBR de SciELO y Dialnet y leve entre los Redalyc y Dialnet.

Palabras clave: SciELO; Redalyc; Dialnet; volumen de búsqueda relativo; Google Trends.

\section{Trends in the searching information on the collections SciELO, Redalyc and Dialnet con- ducted through Google.}

Abstract: The present research was aimed to analyze the trend of information searches on SciELO, Redalic and Dialnet bibliography collections conducted through Google. From the obtained results, it was possible to conclude that in their home country and affiliation of each collection it was observed a high incidence in the following searches: SciELO in Brasil, Redalyc in México and Dialnet in Spain. Throughout the analyzed period, the most sought-after collection was SciELO, although, it was verified a relative search volumen (RSV) both in Redalic and Dialnet searches, this did not befall in the case of SciELO. In none of them has been possible to behold a remarkable landmark. Merely it could be shown a moderate association between the RSV of SciELO and Dialnet and slight between the Redalyc and Dialnet.

Keywords: SciELO; Redalyc; Dialnet; relative search volume; Google Trends

Copyright: ( $\odot 2021$ CSIC. Este es un artículo de acceso abierto distribuido bajo los términos de la licencia de uso y distribución Creative Commons Reconocimiento 4.0 Internacional (CC BY 4.0). 


\section{INTRODUCCIÓN}

La idea de que las poblaciones proporcionen datos sobre sus gustos, la búsqueda de servicios e incluso sobre su enfermedad, a través del comportamiento de búsqueda de información en la Web, ya ha sido explorada en los últimos años (Pelat y otros., 2009; Sanz-Lorente, 2020).

Es conocido que el buscador de Google recopila información relativa al comportamiento de los usuarios que navegan por su dominio $y$, por supuesto, también recoge los términos y expresiones que se utilizan para buscar información en el conocido buscador. Al análisis estadístico de los términos de búsqueda de los usuarios, ya sea en un sitio web particular, en una plataforma de red social o en un buscador web, se le denomina search analytics y forma parte del campo de la cibermetría (Orduña-Malea, 2019).

En el campo de la salud, Eysenbach (2009) acuñó el término de «infodemiología» como un conjunto emergente de métodos de información de salud pública para analizar el comportamiento de la búsqueda, la comunicación y la publicación en Internet. Es decir, observar y analizar el comportamiento basado en la Web para conocer la conducta humana a fin de predecir, evaluar, e incluso prevenir problemas relacionados con la salud que surgen constantemente en la vida cotidiana (Mavragani y otros, 2018).

Así pues, está más que demostrado que Google es un motor de búsqueda que facilita información a cualquier persona, que a través de los resultados obtenidos puede acceder fácilmente a los documentos existentes en la Red. Y, aunque no es el útil más conocido de Google, Google Trends (GT) es una herramienta de acceso libre, que informa del volumen de búsquedas realizadas por los usuarios de todo el mundo para exponer qué tan frecuentemente se busca un término y en qué lugares. Los datos de búsqueda en Internet pueden proporcionar información valiosa sobre los patrones de comportamiento de la población (Nuti y otros, 2014; Sanz-Lorente y Wanden-Berghe, 2018).

En los últimos años, los datos obtenidos a través de Google Trends han sido utilizados en investigaciones de diversos ámbitos y con distintas finalidades. Estos datos se han tomado como indicadores para estimar la incidencia de enfermedades (Sanz-Lorente y otros, 2019), para predecir niveles de desempleo (Redondo Caballero, 2013) o para analizar proyecciones de ventas (Belapatiño y Crispin, 2016).

Ahora bien, para los investigadores las revistas académico-científicas son uno de los principales canales de comunicación y difusión de los resultados de la investigación y las revistas más solicitadas depen- den, en gran medida, de su calidad y su visibilidad. Cuanta más visibilidad tiene una revista, más interés despierta y, por ende, mayor cantidad de trabajos recibe para su publicación. Las bases de datos y otros repertorios de difusión de la literatura publicada desempeñan un rol central en este contexto, como vehículos que contribuyen a incrementar la visibilidad de las revistas y artículos, en tanto que la calidad es el filtro de editores y distribuidores para su inclusión en dichas fuentes (Villamón y otros, 2005).

Las colecciones digitales conservan y preservan la producción científica, favorecen su difusión, accesibilidad y utilización, además de aumentar su visibilidad e impacto en la comunidad científica. Por tanto, las colecciones de revistas serán foco de interés de profesionales e investigadores y en el ámbito iberoamericano hay tres que destacan sobremanera: SciELO, Redalyc y Dialnet.

Las tres plataformas analizadas son portales de revistas científicas de ámbito ibero-latinoamericano y carácter multidisciplinar, pero presentan características diferenciadoras.

SciELO y Redalyc surgen como una respuesta de la Región a la escasa visibilidad de sus revistas en las principales bases de datos, y el bajo impacto de la investigación realizada en estos países en los circuitos principales de la ciencia.

SciELO nace en el año 1998, en Brasil, bajo tres premisas básicas: aumentar la visibilidad de las revistas brasileñas mediante la publicación electrónica y el libre acceso a los textos, proporcionar indicadores bibliométricos sobre la ciencia producida en la región y mejorar la calidad de sus revistas científicas. El modelo, poco a poco, se fue extendiendo por la región y hoy SciELO funciona como una red descentralizada de hemerotecas científicas que abarca 14 países y tres continentes (http:// www.scielo.org). En conjunto, la red indiza más de 1.440 revistas y da acceso a más de 700.000 artículos, con un incremento medio de más de 40.000 artículos por año (Packer y otros, 2014; Packer y Meneghini, 2015).

Algunos años después, en 2003, la Universidad Autónoma de México, pone en marcha el sitio Redalyc (https://www.redalyc.org/) bajo unas premisas muy similares a las de SciELO, pero funcionando como una única hemeroteca virtual de libre acceso, no como una red, y más centrada en reunir la producción más relevante de las Ciencias Sociales, mientras que SciELO nació con un carácter biomédico. Hoy ambas plataformas son multidisciplinares y presentan cifras muy similares, Redalyc cuenta con 1360 revistas y 670.000 artículos (Aguado-López y otros, 2003). 
Dialnet tiene un origen distinto, nace en el año 2001 , bajo otros planteamientos. Se crea con la idea de dar un servicio de alertas bibliográficas accesible a cualquier usuario, poco a poco se convierte en una hemeroteca virtual y un servicio de alojamiento de textos, todo ello con vocación integradora en el sentido de almacenar distintos tipos de documentos (científicos, técnicos, divulgativos, etc.), algunos de manera referencial y otros a texto completo. Dialnet recoge artículos de revistas, tesis, actas de congresos, capítulos de libros...etc., publicados en España o en español en cualquier país. En este caso con claro predominio de revistas de Humanidades y Ciencias Sociales (Mateo, 2015).

Desde hace años, los motores de búsqueda son el punto de partida para la navegación web, permitiendo al usuario encontrar contenidos en Internet utilizando una serie de términos. Google, fundado 1997, no necesita presentación, es el motor de búsqueda más utilizado en el mundo, con una cuota de mercado que rebasa ampliamente a otros buscadores como Baidu o Yandex (los más usados en China y Rusia respectivamente) (Amazon Company, sin fecha, Net Market Share, 2020). En este sentido, es conocido que una gran mayoría de los estudiantes universitarios norteamericanos empiezan sus búsquedas de información en buscadores web, en vez de utilizar algún recurso de la biblioteca (Ávila-García y otros, 2015). Actitud que es compartida por muchas personas que, por olvido de la dirección electrónica (Uniform Resource Locator), o simplemente por comodidad, utilizan Google como puerta de inicio de su búsqueda.

Con este potencial, como indicó Orduña-Malea (2019), GT puede ser utilizado en tareas de newcasting (predecir el presente, es decir, pronosticar valores que están sucediendo al mismo tiempo que se generan los datos), y de forecasting (predicción de tendencias futuras). Así, esta herramienta puede ayudar a los responsables de estas 3 plataformas a conocer los productos más buscados, cuáles son las nuevas tendencias, y qué necesidades están demandando los usuarios (Morán, 2016).

En este contexto, el objetivo de este estudio fue analizar la tendencia de las búsquedas de información y los hitos, sobre estas tres colecciones, que se realizan a través de Google.

\section{MATERIAL Y MÉTODO}

\subsection{Diseño}

Estudio descriptivo y correlacional de las tendencias de búsqueda de información sobre SciELO, Redalyc y Dialnet, a través de Google y en todo el mundo.

\subsection{Fuente de obtención de la información}

Los datos sobre búsqueda de información se obtuvieron de la consulta directa, mediante acceso online, a Google Trends (https://trends.google.es/).

El periodo a estudio fue del 1 de enero de 2004 hasta el 31 de diciembre de 2019. El ámbito fue todo el mundo y en todas las categorías. El periodo estudiado es común a las tres colecciones, puesto que SciELO se crea en el año 1998, Dialnet en 2001 y Redalyc surge en el año 2002. La fecha de consulta y recolección de los datos fue el 17 de febrero de 2020.

\subsection{Tema de búsqueda}

Las búsquedas se realizaron con las palabras «SciELO», «Redalyc»y «Dialnet» como «Tema»y tomando los resultados para todo el mundo y en todas las categorías.

Hay que tener en cuenta que en esta herramienta la consulta utilizando un "Término de búsqueda" muestra las coincidencias de todos los términos de la consulta en el idioma especificado (Ejemplo: si se busca "salud" se obtendrán también resultados sobre "salud pública", "ciencias de la salud", etc.). En cambio, si se utiliza el vocablo como "Tema" se obtendrán los resultados de los términos que comparten el mismo concepto en cualquier idioma. (Ejemplo: si se busca "Londres", la búsqueda incluye resultados de temas como los siguientes "capital del Reino Unido", "London" o "London city", entre otros).

\subsection{Obtención y almacenamiento de los datos}

Los resultados obtenidos fueron descargados en formato normalizado CSV (comma-separated values) que permitió su almacenamiento posterior en un archivo Excel.

Las gráficas GT representan con cuánta frecuencia se realiza una búsqueda de un término particular en varias regiones del mundo y en varios idiomas. El eje horizontal de la gráfica representa el tiempo (desde el año 2004), y el eje vertical representa la frecuencia, como volumen de búsqueda relativo (VBR), con la que se ha buscado el término globalmente, permitiendo al usuario comparar el VBR entre dos o más términos (actualmente un máximo de 5).

El control de la calidad de esta información se efectuó mediante dobles tablas, corrigiendo las posibles inconsistencias mediante la consulta con la tabla original descargada. Para el análisis estadístico se utilizó el programa Statistical Package for the Social Sciences (SPSS) para Windows versión 25.0. 


\subsection{Variables a estudio}

- Volumen de búsqueda relativo (VBR): resultado mensual ofrecido por Google Trends cuyos valores se normalizan en una escala de 0 (un volumen de búsqueda relativo inferior al $1 \%$ del volumen máximo) a 100 (el volumen de búsqueda relativo alcanza su máximo). Por ejemplo, un VBR $=25$, representa el $25 \%$ de la proporción de búsqueda observada más alta durante el período a estudio.

- Hito: acontecimiento puntual y destacado en el VBR.

\subsection{Análisis de los datos}

Se obtuvieron las medidas de tendencia central (media y su desviación estándar, la mediana, la amplitud intercuartílica (AIQ) y el máximo y mínimo) para describir las variables a estudio. Se analizó la existencia de asociación entre las variables cuantitativas para comprobar la significación de la diferencia de medias para muestras independientes, utilizando el test de la $\mathrm{t}$ de Student. La evolución temporal de las tendencias de búsqueda se examinó mediante el análisis de regresión, calculándose el coeficiente de determinación $\left(R^{2}\right)$. Para obtener la relación entre variables cuantitativas se usó el coeficiente de correlación de Pearson (medida de dependencia lineal entre dos variables aleatorias cuantitativas).

El nivel de significación utilizado en todos los contrastes de hipótesis fue $a \leq 0,05$.

Para analizar la evolución del VBR mensual se segmentó el período de estudio en dos épocas iguales: $1^{\text {a }}$ época: del 1 enero de 2004 al 30 junio de 2011 y $2^{a}$ época: del 1 julio de 2011 al 31 de diciembre de 2019.

Para todos los cálculos relacionados con el VBR se tuvieron en cuenta los valores mensuales de cada uno de los años; ver Anexo I.

\section{RESULTADOS}

A partir de la consulta efectuada se obtuvieron los valores mensuales de los VBR para los temas «SciELO», «Redalyc» y «Dialnet» obtenidos de Google Trends, ver Anexo I, y las correspondientes

Figura 1. Tendencia de búsqueda en Google Trends para los temas SciELO, Redalyc y Dialnet (desde el 1 ene de 2004 a 31 dic de 2019).

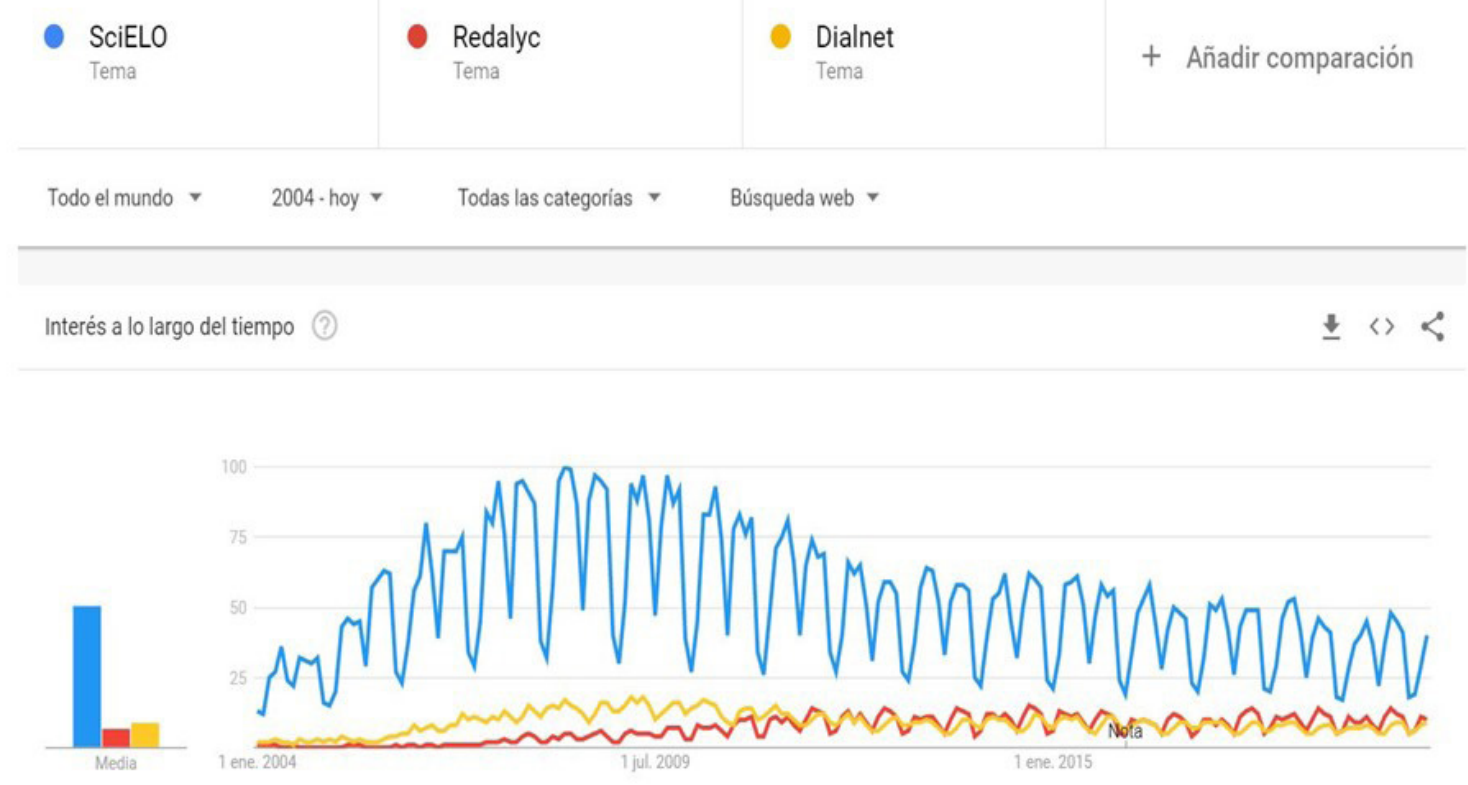

\begin{tabular}{|c|c|c|c|c|c|c|c|c|c|c|c|c|c|c|c|}
\hline \multicolumn{16}{|c|}{$\begin{array}{l}\text { Sumatorio de los volúmenes relativos de búsqueda mensuales, según año, para cada de los temas «SciELO», «Redalyc» y «Dialnet», obtenidos de } \\
\text { Google Trends }\end{array}$} \\
\hline Año & SciELO & Redalyc & Dialnet & Año & SciELO & Redalyc & Dialnet & Año & SciELO & Redalyc & Dialnet & Año & SciELO & Redalyc & Dialnet \\
\hline 2004 & & & & 20 & & & & 6 & & 12 & 89 & 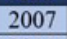 & & -5 & 134 \\
\hline & & & & 20 & & & 175 & & & r & 156 & & & 3 & 32 \\
\hline 2012 & & 120 & 107 & 2013 & 579 & 115 & 97 & 2014 & 56 & 125 & 107 & 2015 & 552 & 102 & 106 \\
\hline 2016 & 492 & 102 & 92 & 2017 & 484 & 112 & 91 & 2018 & 454 & 118 & 85 & 2019 & 433 & 118 & 88 \\
\hline
\end{tabular}


Figura 2. Desglose comparativo por país de los resultados globales de los temas SciELO, Redalyc y Dialnet (desde el 1 ene de 2004 a 31 dic de 2019), obtenidos de Google Trends.

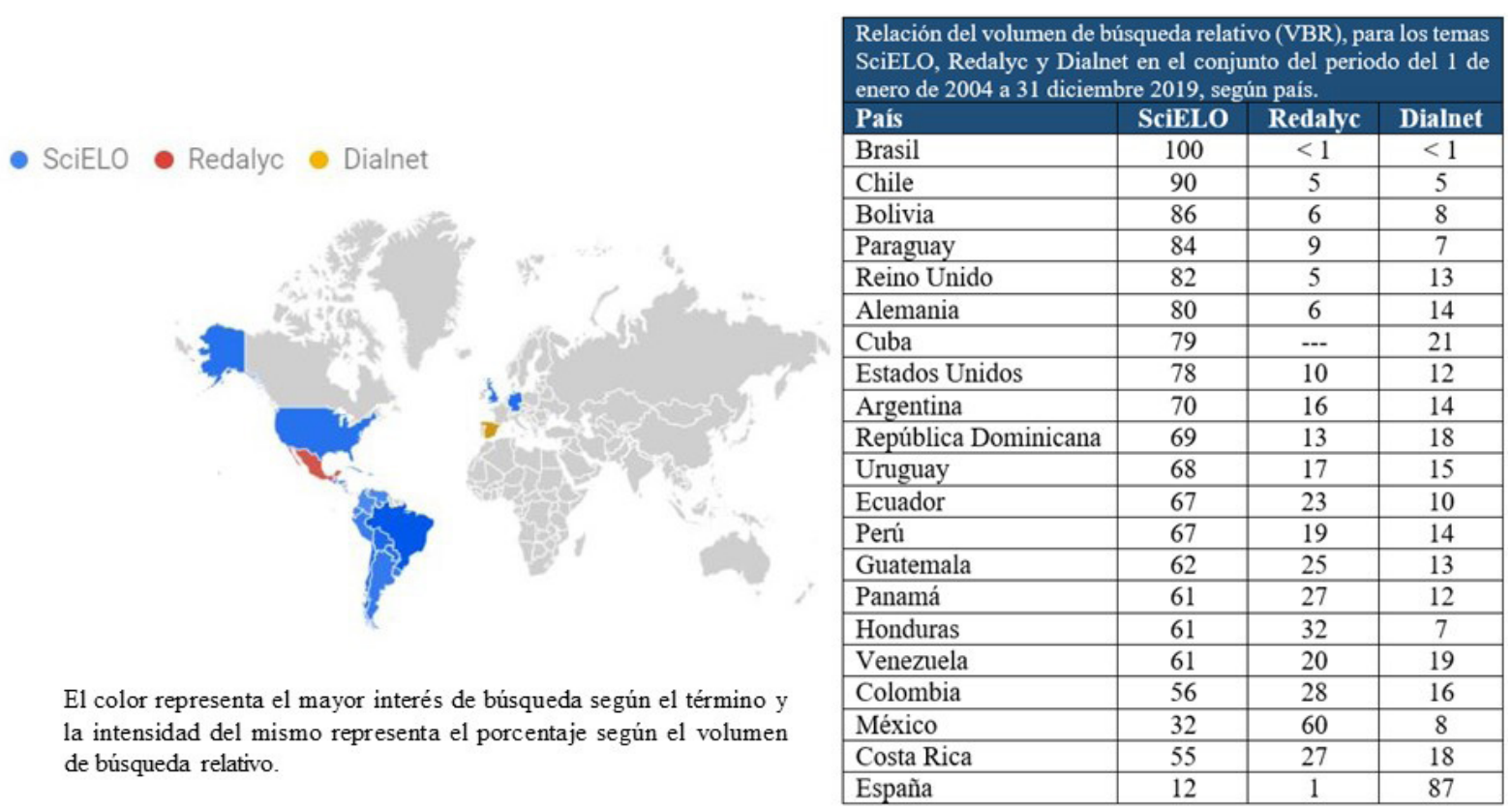

tendencias de búsqueda para cada una de las plataformas; ver captura de pantalla en la Figura 1.

Al mismo tiempo, y tomando la imagen ofrecida por esta herramienta, se obtuvo el desglose comparativo de los resultados globales para los 3 temas a estudio, observándose el interés principal según país; ver captura de pantalla en la Figura 2.

\subsection{Hitos}

El principal hecho puntual en el VBR se produjo en la consulta sobre «SciELO» en abril de 2008 $(\mathrm{VBR}=100)$. La relación de los VBR en este hito entre «SciELO» versus «Redalyc» versus «Dialnet» fue: $100 / 5 / 17$

\subsection{Volumen de búsqueda relativo}

Los estadísticos de los VBR para los términos analizados pueden consultarse en la Tabla 1, sien- do la correspondencia media de los VBR entre «SciELO» versus «Redalyc» versus «Dialnet» de $51,18 / 6,73 / 8,78$.

La tendencia de VBR para «SciELO» presentó un gráfico con progresión decreciente y ajuste a un modelo lineal $\left(R^{2}=0,05 ; p=0,001\right)$; para «Redalyc» se obtuvo una evolución moderadamente creciente con ajuste lineal $\left(R^{2}=0,58 ; p<0,001\right)$; y Dialnet mostró un suave progreso exponencial creciente $\left(R^{2}=0,06 ; p=0,001\right)$; ver Figura 3 .

Al analizar los datos del VBR para «SciELO», según época, se constató que existían diferencias significativas ( $p<0,001$ ) a favor de la primera época (valores de las medias: 59,19 versus 44,11). Igualmente, se mostraron diferencias significativas $(p=0,021)$ a favor de la primera época en los VBR para «Dialnet» (valores de las medias: 9,49 versus 8,16$)$. Por el contrario, los valores del VBR

Tabla I. Estadísticos del Volumen de Búsqueda Relativo (VBR) de los temas «SciELO», «Redalyc» y «Dialnet», obtenidos de Google Trends

\begin{tabular}{|l|l|r|r|r|c|}
\hline Término & \multicolumn{1}{|c|}{ Media } & Mediana & Máximo & Mínimo & AIQ \\
\hline SciELO & $51,18 \pm 1,60$ & 48,50 & 100 & 12 & 31 \\
\hline Redalyc & $6,73 \pm 0,31$ & 6 & 15 & 0 & 8 \\
\hline Dialnet & $8,78 \pm 0,27$ & 9 & 18 & 1 & 5 \\
\hline AIQ = Amplitud Intercuartílica
\end{tabular}


Figura 3. Evolución temporal de los resultados mensuales para SciELO, Redalyc y Dialnet (desde el 1 ene de 2004 a 31 dic de 2019), obtenidos de Google Trends.

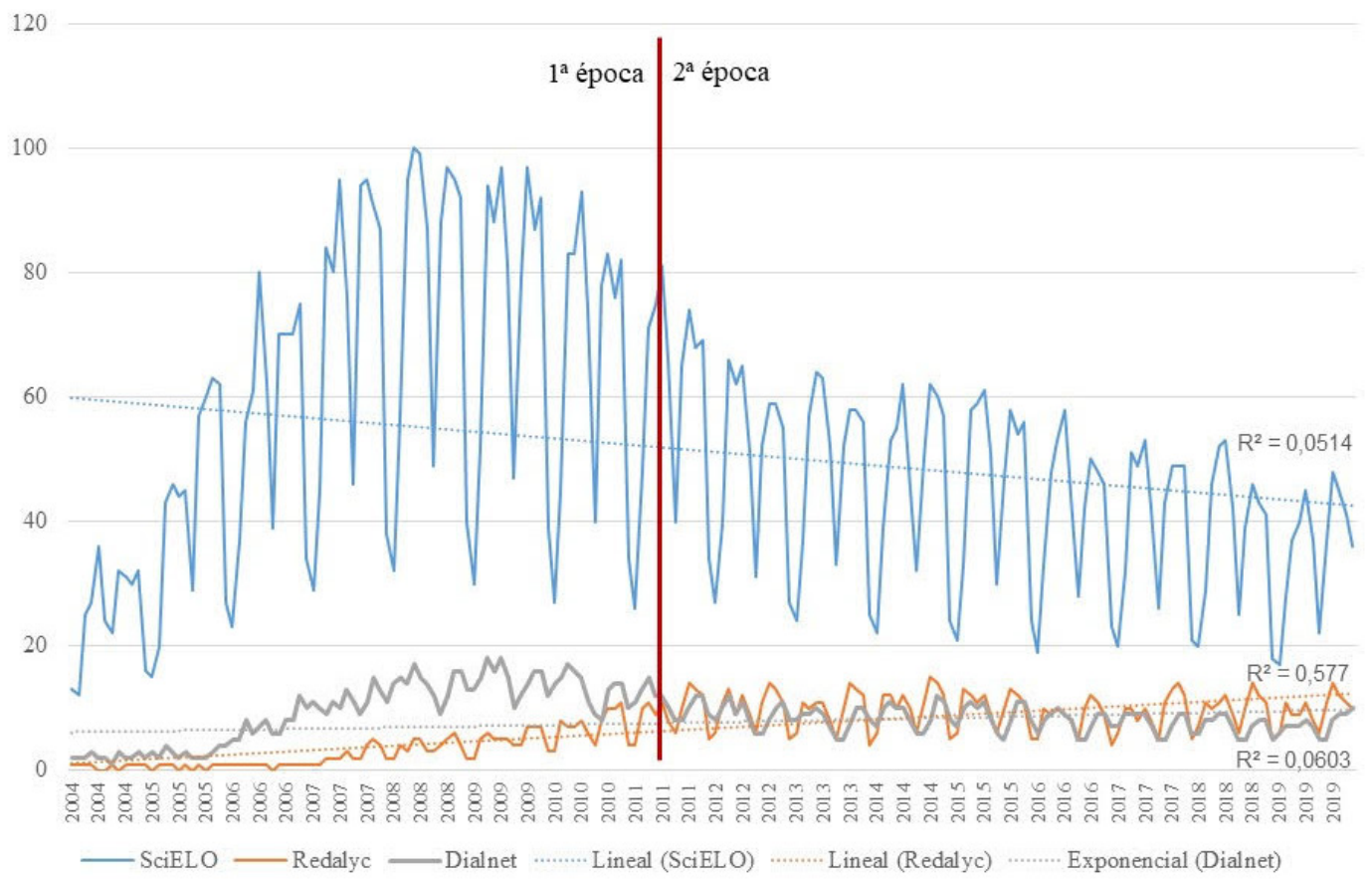

Figura 4. Comparación de las medias del volumen de búsqueda relativo (VBR), para los temas SciELO, Redalyc y Dialnet, obtenidos de Google Trends, según época a estudio (1a época: 1 de enero de 2004 a 30 de junio de 2011; 2a época de 1 de julio de 2011 a 31 de diciembre de 2019).
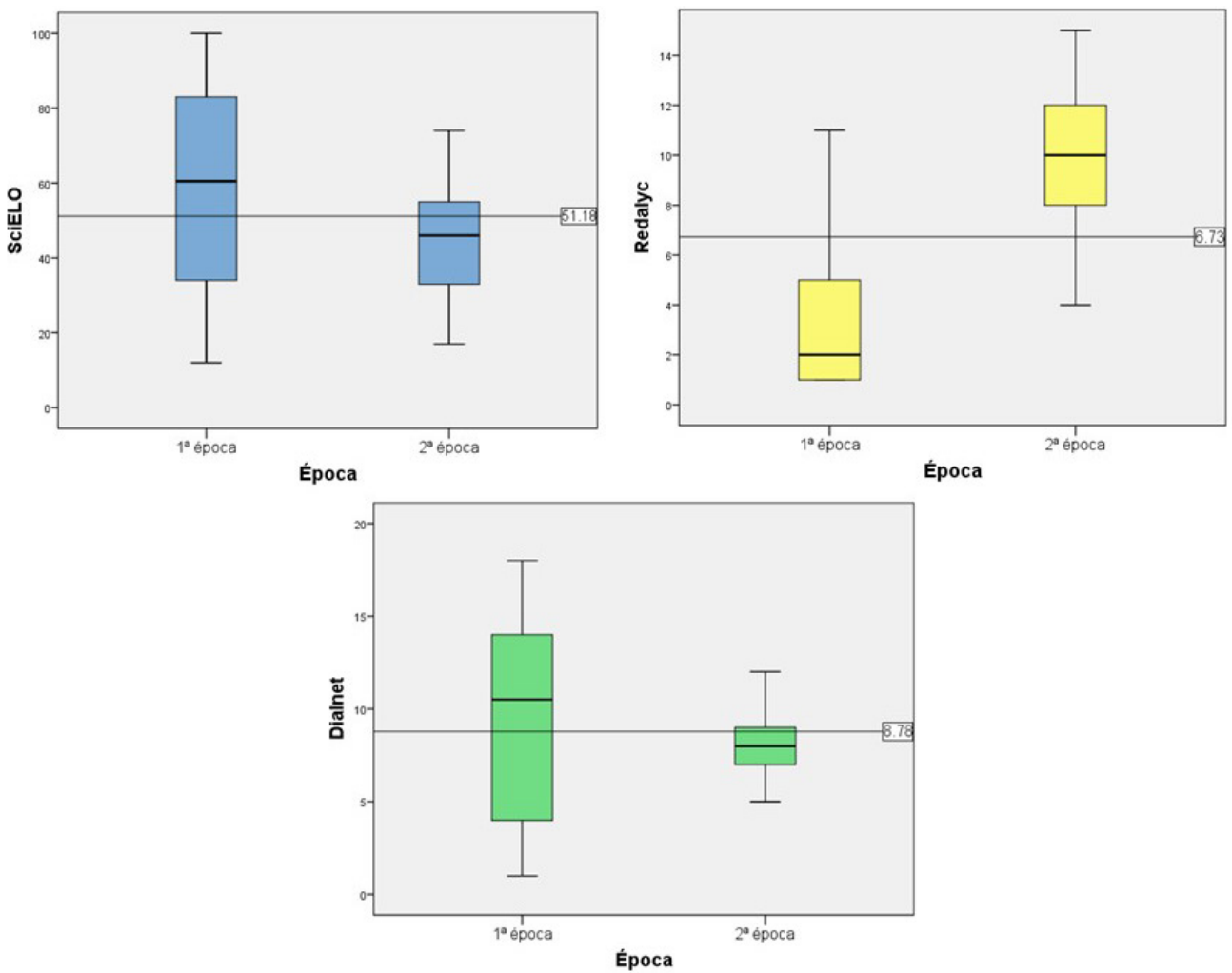
para «Redalyc» dieron diferencias significativas ( $p$ $<0,001$ ) a favor de la segunda época (valores de las medias: 3,46 versus 9,71); ver Figura 4.

\subsection{Asociación entre los diferentes VBR}

La correlación observada entre los VBR de los temas «SciELO»y «Redalyc» no mostró asociación entre ellos $(R=0,13 ; p=0,076)$. Por el contrario, si existió buena relación entre los VBR de los temas «SciELO»y «Dialnet» $(R=0,66, p<0,001)$ y leve asociación entre los VBR de temas «Redalyc» $y \ll$ Dialnet» $(R=0,26 ; p<0,001)$.

\section{DISCUSIÓN}

A la vista de los resultados se puede deducir que las tendencias de los motores de búsqueda son una herramienta que puede integrar la vigilancia, en tiempo real, de las necesidades de información de la población. Así, Anderegg y Goldsmith, en 2014, ya afirmaban que Google Trends era una herramienta que se reconocía como indicador sólido y válido para la predicción del patrón de comportamiento en la búsqueda de información (Anderegg y Goldsmith, 2014).

Pero, es importante dejar constancia de que este estudio no pretendió comparar la cobertura de estas plataformas, ni discutir la validez de los contenidos que indizan. El valor del análisis de los VBR propuesto está en conocer cuáles son las búsquedas de los usuarios, de todo el mundo, en un determinado periodo de tiempo y ver cómo evolucionan los comportamientos sociales.

En el desglose comparativo por país de los resultados globales, quedó patente el predominio de cada una de estas colecciones bibliográficas en su país de origen y filiación, situación que confirmó la conjetura de partida y por la correspondencia media de los VBR. Igualmente, era esperable que SciELO tuviera un claro predominio en Latinoamérica. Según Ugarte (2004), la indización en SciELO constituyó, y constituye, la principal herramienta para que esta producción científica saliera de la literatura gris y accediera a los índices de medición bibliométrica clásicos, fundamentalmente por las aportaciones de Brasil, Argentina y Chile (Aguirre-Pitol y otros, 2013).

La evolución temporal de los resultados mensuales para las búsquedas sobre las tres colecciones también mostró un mayor VBR a favor de SciELO, si bien ninguna de las colecciones presentó un claro hito que permitiera fijar un hecho concreto, si acaso el máximo que se dio en 2008 , año que puede relacionarse con el décimo aniversario de la creación de SciELO. Lo que más Ilama la aten- ción es la forma en diente de sierra que se observa principalmente en el VBR de SciELO, típico de los gráficos de las enfermedades epidemiológicas, con máximos que se centran entre abril y mayo de cada año y mínimos en diciembre y enero. Una posible explicación de esta tendencia podría relacionarse con la actividad académica que durante parte de diciembre y enero disminuye su actividad. Ahora bien, los dientes de sierra también son una visualización que permite reconocer gráficamente la relación entre turnos de un diálogo, una tipología de figuras interaccionales que recoge las diferentes estructuras que se producen en la toma de turnos de la conversación coloquial (sea verbal, escrita o digital) (Espinosa Guerri, 2019).

La tendencia observada en el VBR de Redalyc y Dialnet sigue el progresivo incremento en la producción científica de las revistas del área latinoamericana (Bojo-Canales y Sanz-Valero, 2019). El VBR observado en estas dos colecciones, no alcanzó la llamada «explosión de la información» que lleva a modelos de crecimiento exponencial (Sanz-Valero y Wanden-Berghe, 2017), ya que sus crecimientos solo pueden ser calificados de leves o, como mucho, moderados. De todos modos, estas tendencias no se basan en datos de crecimiento o decrecimiento absolutos, pues el indicador estudiado, VBR, como su mismo nombre indica ofrece valores relativos.

Situación diferente son los VBR para la colección SciELO; la evolución temporal de los VBR dio, sorprendentemente, un resultado de progresión decreciente contrario a lo esperado, teniendo en cuenta el incremento en la producción científica que recoge esta colección (Bojo-Canales y Sanz-Valero, 2019). Una posible explicación sea que al ser una colección ampliamente conocida $y$, por tanto, como se recoge asimismo en las limitaciones de este estudio, muchos profesionales e investigadores accedan directamente desde la homepage de SciELO sin necesidad de localizarla a través de Google. Aun así, hace ya tiempo que los contenidos de las plataformas, como las analizadas aquí, son "recolectados" por Google, de modo que, ante cualquier búsqueda de información un usuario podría encontrar, entre los resultados ofrecidos, un registro que estuviera recogido por una de estas 3 plataformas.

La observación de una moderada correlación entre los VBR de SciELO y Dialnet y leve entre los Redalyc y Dialnet se debería, principalmente, a que en la mayoría de las veces cuando se busca el término correspondiente a una de estas colecciones no se hace con los otros dos. Por otra parte, puede no existir una misma motivación para buscar en las tres ya que no todas las revistas están incluidas a la vez en las tres ni existe un equilibrio temático; 
por ejemplo, Redalyc tiene un mayor sesgo hacia las Ciencias Sociales mientras que SciELO lo tiene hacia las Ciencias de la Salud (Miguel, 2011).

\section{LIMITACIONES}

De acuerdo con Johnson y Mehta (2014), el que Google Trends no facilite datos de uso real e intervalos de tiempo más precisos, disminuye la capacidad de pronóstico. Hay que tener presente que se trataba de un análisis de datos ecológicos y los hallazgos pudieron no ser representativos a nivel individual. Además, como sugieren Cervellin y otros (2017), los resultados que se obtienen mediante esta herramienta pueden estar influenciados por el interés de los medios.

Un hecho importante es que GT ofrece valores relativos, pero no frecuencias absolutas (número total de búsquedas), lo que disminuye la capacidad de pronóstico y de análisis estadísticos más reales. Además, se echa en falta una mayor transparencia, ya que no existe información sobre los métodos específicos que Google utiliza para pronosticar las tendencias, los cuales no han sido divulgados por la compañía (Orellano y otros, 2015).

Por otro lado, puede presentarse un sesgo debido a que la búsqueda se realizó solo en Google y no se contabilizaron las realizadas en otros buscadores o incluso mediante la consulta a las hemerotecas. Más aún, muchos profesionales acceden directamente a estas colecciones sin pasar, previamente, por la búsqueda utilizando Google, por lo que estas búsquedas directas, que determinan el uso y utilidad de dichos portales, no quedan reflejadas en este artículo. No obstante, el estudio de Gardner e Inger (2018), sobre los hábitos de búsqueda de los lectores de literatura científica, señalaba como resultado importante que mientras que las bases bibliográficas seguían siendo la fuente más relevante, su importancia venía disminuyendo desde 2008 , perdiendo posiciones frente a los motores de búsqueda, las redes sociales y los servicios de agregadores.

\section{REFERENCIAS}

Aguado-López, E.; Sandoval Forero, E.; Chávez-Ávila, S. (2003). La ciencia perdida y las nuevas tecnologías de divulgación del conocimiento: el proyecto Redalyc. Revista Educación y Ciencia, 7(13), 11-39. http://hdl. handle.net/20.500.11799/6618

Aguirre-Pitol, M. Á.; Leal-Arriola, M.; Martínez-Domínguez, N. D. (2013). Cápsulas de Investigación n³Análisis comparativo de la cobertura de SciELO y Redalyc [en línea]. Disponible en: https://bit.ly/3dvvWHm [Fecha de consulta: 15 febrero de 2020]

Amazon Company. (s. f.). Alexa, The top 500 sites on the Web [en línea]. Disponible en: http://www.alexa.com/ topsites [Fecha de consulta: 17/05/2020]

\section{CONCLUSIONES}

El uso de una herramienta como Google Trends puede proporcionar una valiosa información a los gestores y administradores de estas plataformas, sobre las búsquedas no dirigidas ni especializadas, lo que puede ser muy útil para implementar estrategias de visibilización de sus contenidos. Estas tácticas deben estar fundamentadas en datos de investigación que proporcionen la información adecuada para desarrollar el mejor material posible. Además, pueden conocer el interés de la población en unos determinados temas y en un momento determinado.

En este estudio se observó un claro predomino en las búsquedas en su país de origen y filiación de cada una de las colecciones: SciELO en Brasil, Redalyc en México, y Dialnet en España. La colección más buscada fue SciELO en todo el periodo de tiempo analizado y si bien se constató un crecimiento en el VBR tanto en las búsquedas sobre Redalyc y Dialnet, no sucedió así en el caso de SciELO. Y, en ninguna de ellas se pudo observar un hito claramente destacable. Solo se pudo demostrar una moderada asociación entre los VBR de SciELO y Dialnet y leve entre los Redalyc y Dialnet.

\section{AGRADECIMIENTOS}

A Habiba Chbab, con maestría en inglés y español para fines específicos y estudiante de doctorado en Traducción profesional y audiovisual (rama de investigación: traducción médica), por su colaboración en la traducción al inglés del resumen de este artículo.

\section{ACKNOWLEDGEMENTS}

To Habiba Chbab, master's degree in English and Spanish for Specific Purposes and doctoral student in Professional and Audiovisual Translation (Research branch: medical translation), for her collaboration in the English translation of the abstract of this article.

Anderegg, W. R. L.; Goldsmith, G. R. (2014). Public interest in climate change over the past decade and the effects of the 'climategate' media event. Environmental Research Letters, 9(5), 054005. https://doi. org/10.1088/1748-9326/9/5/054005

Ávila-García, L.; Ortiz-Repiso, V.; Rodríguez-Mateos, D. (2015). Herramientas de descubrimiento: ¿una ventanilla única? Revista Española de Documentación Científica, 38 (1):e077. http://dx.doi.org/10.3989/redc.2015.1.1178

Belapatiño, V.; Crispin, Y. (2016). Perú-Búsquedas en internet $y$ proyecciones de ventas de departamentos en Lima [en línea]. Disponible en: https://bit.ly/3bkLo7i [Fecha de consulta: 15 febrero de 2020] 
Bojo-Canales, C.; Sanz-Valero, J. (2019). Las revistas de ciencias de la salud de la red SciELO: Un análisis de su visibilidad en el ámbito internacional. Revista Española de Documentación Científica, 42(4), 245. https://doi. org/10.3989/redc.2019.4.1629

Cervellin, G.; Comelli, I.; Lippi, G. (2017). Is Google Trends a reliable tool for digital epidemiology? Insights from different clinical settings. Journal of Epidemiology and Global Health, 7(3), 185-189. https://doi. org/10.1016/j.jegh.2017.06.001

Espinosa Guerri, G. (2019). Dibujar la conversación en ELE: La enseñanza de la toma de turnos del español. Foro de profesores de $E / L E, 15$. https://doi. org/10.7203/foroele.15.14860

Eysenbach, G. (2009). Infodemiology and infoveillance: Framework for an emerging set of public health informatics methods to analyze search, communication and publication behavior on the Internet. Journal of Medical Internet Research, 11(1), e11. https://doi. org/10.2196/jmir.1157

Gardner, T.; Inger, S. (2018). How readers discover content in scholarly publications [en línea]. Disponible en: https://bit.ly/2Ufk3xL [Fecha de consulta: 15 febrero de 2020]

Johnson, A. K.; Mehta, S. D. (2014). A comparison of Internet search trends and sexually transmitted infection rates using Google trends. Sexually Transmitted Diseases, 41(1), 61-63. https://doi.org/10.1097/ OLQ.0000000000000065

Mateo, F. (2015). Producción científica en español en humanidades y ciencias sociales: Algunas propuestas desde Dialnet. El profesional de la Información, 24,(5), 509-515. https://doi.org/10.3145/epi.2015.sep.01

Mavragani, A.; Ochoa, G.; Tsagarakis, K. P. (2018). Assessing the methods, tools, and statistical approaches in Google Trends research: Systematic review. Journal of Medical Internet Research, 20(11), e270. https:// doi.org/10.2196/jmir.9366

Miguel, S. (2011). Revistas y producción científica de América Latina y el Caribe: Su visibilidad en SciELO, RedALyC y SCOPUS. Revista Interamericana de Bibliotecología, 34(2), 187-199.

Morán, J. (2016). Google Trends: una nueva herramienta para la predicción económica - Aplicaciones para completar el análisis econométrico tradicional [en línea]. Disponible en: http://hdl.handle.net/10908/12083 [Fecha de consulta: 10 mayo de 2020]

Net Market Share. (2020). Search Engine Market Share [en línea]. Disponible en: https://bit.ly/3fYLm8k [Fecha de consulta: 17/05/2020]

Nuti, S. V.; Wayda, B.; Ranasinghe, I.; Wang, S.; Dreyer, R. P.; Chen, S. I.; Murugiah, K. (2014). The use of google trends in health care research: A systematic review. PloS One, 9(10), e109583. https://doi. org/10.1371/journal.pone.0109583

Orduña-Malea, E. (2019). Google Trends: Analítica de búsquedas al servicio del investigador, del profesio- nal y del curioso de la información. Anuario ThinkEPI, 13, e13inf01. https://doi.org/10.3145/thinkepi.2019. e13inf01

Orellano, P.W.; Reynoso, J.I.; Antman, J.; Argibay, O. (2015). Uso de la herramienta Google Trends para estimar la incidencia de enfermedades tipo influenza en Argentina. Cadernos de Saude Publica, 31(4):691700. https://doi.org/10.1590/0102-311x00072814

Packer. A.L.; Cop, N.; Luccisano, A.; Ramalho, A.; Spinak, E. (2014). SciElO: 15 Años de Acceso Abierto - Un estudio analítico sobre Acceso Abierto y comunicación científica. Paris, Francia: UNESCO. http://dx.doi. org/10.7476/9789233012370

Packer, A.L.; Meneghini, R. Contribución de SciELO a la Globalización de la Ciencia [en línea]. Disponible en: https://bit.ly/2ZIF6St [Fecha de consulta 17/05/2020]

Pelat, C. ; Turbelin, C.; Bar-Hen, A.; Flahault, A.; Valleron, A.-J. (2009). More diseases tracked by using Google Trends. Emerging Infectious Diseases, 15(8), 13271328. https://doi.org/10.3201/eid1508.090299

Redondo Caballero, J. (2013). Uso de Google Trends para predecir el nivel y la estructura del desempleo en España [tesis doctoral] [en línea]. Universitat Politècnica de València, Facultad de Administración y Dirección de Empresas. Disponible en: http://hdl.handle. net/10251/31028 [Fecha de consulta: 15 febrero de 2020]

Sanz-Lorente, M. (2020). Tendencias temporales de los patrones de búsqueda de información sobre servicio de asistencia sanitaria domiciliaria en España. Hospital a Domicilio, 4(1), 15-23. https://doi.org/10.22585/ hospdomic.v4i1.95

Sanz-Lorente, M.; Sanz-Valero, J.; Wanden-Berghe, C. (2019). Tendencias temporales de los patrones de búsqueda de información sobre VIH/sida en España. Revista Española de Comunicación Salud, Supl 2, S52-S60. https://doi.org/10.20318/recs.2019.4554

Sanz-Lorente, M.; Wanden-Berghe, C. (2018). Tendencias temporales de los patrones de búsqueda de información sobre cuidado domiciliario "Home Care" u hospitalario "Hospital Care" a través de Google. Hospital a Domicilio, 2(3), 93-99. https://doi.org/10.22585/hospdomic.v2i3.47

Sanz-Valero, J.; Wanden-Berghe, C. (2017). Análisis bibliométrico de la producción científica, indizada en MEDLINE, sobre los servicios de salud proporcionados por las unidades de hospitalización a domicilio. Hospital a Domicilio, 1(1), 21-34. https://doi.org/10.22585/ hospdomic.v1i1.3

Ugarte P, F. (2004). La edición de revistas científicas en Latinoamérica. Revista Chilena de Pediatría, 75(6), 509-511. https://doi.org/10.4067/S037041062004000600001

Villamón, M.; Devis-Devis, J.; Valenciano-Valcárcel, ]. (2005). Análisis de la visibilidad de las revistas científico-técnicas españolas de ciencias de la actividad física y el deporte. Revista de Psicología del Deporte, 14(2), 253-267. 


\section{ANEXO I}

Valores mensuales de los volúmenes de búsqueda relativos para los temas «SciELO», «Redalyc»y «Dialnet», obtenidos de Google Trends

\begin{tabular}{|c|c|c|c|c|c|c|c|c|c|}
\hline \multirow{2}{*}{ Año } & \multirow{2}{*}{ Mes } & \multicolumn{3}{|c|}{ Volumen de búsqueda relativo } & \multirow{2}{*}{ Año } & \multirow{2}{*}{ Mes } & \multicolumn{3}{|c|}{ Volumen de búsqueda relativo } \\
\hline & & SciELO & Redalyc & Dialnet & & & Scielo & Redalyc & Dialnet \\
\hline \multirow{12}{*}{2004} & 1 & 13 & 1 & 2 & \multirow{12}{*}{2007} & 1 & 1 & 29 & 1 \\
\hline & 2 & 12 & 1 & 2 & & 2 & 2 & 45 & 1 \\
\hline & 3 & 25 & 1 & 2 & & 3 & 3 & 84 & 2 \\
\hline & 4 & 27 & 1 & 3 & & 4 & 4 & 80 & 2 \\
\hline & 5 & 36 & 1 & 2 & & 5 & 5 & 95 & 2 \\
\hline & 6 & 24 & 1 & 2 & & 6 & 6 & 76 & 3 \\
\hline & 7 & 22 & 1 & 1 & & 7 & 7 & 46 & 2 \\
\hline & 8 & 32 & 1 & 3 & & 8 & 8 & 94 & 2 \\
\hline & 9 & 31 & 1 & 2 & & 9 & 9 & 95 & 4 \\
\hline & 10 & 30 & 1 & 2 & & 10 & 10 & 91 & 5 \\
\hline & 11 & 32 & 1 & 3 & & 11 & 11 & 87 & 4 \\
\hline & 12 & 16 & 1 & 2 & & 12 & 12 & 38 & 2 \\
\hline \multirow{12}{*}{2005} & 1 & 1 & 15 & 1 & \multirow{12}{*}{2008} & 1 & 32 & 2 & 14 \\
\hline & 2 & 2 & 20 & 1 & & 2 & 57 & 4 & 15 \\
\hline & 3 & 3 & 43 & 1 & & 3 & 95 & 3 & 14 \\
\hline & 4 & 4 & 46 & 1 & & 4 & 100 & 5 & 17 \\
\hline & 5 & 5 & 44 & 1 & & 5 & 99 & 5 & 15 \\
\hline & 6 & 6 & 45 & 1 & & 6 & 87 & 3 & 14 \\
\hline & 7 & 7 & 29 & 1 & & 7 & 49 & 3 & 12 \\
\hline & 8 & 8 & 57 & 1 & & 8 & 88 & 4 & 9 \\
\hline & 9 & 9 & 60 & 1 & & 9 & 97 & 5 & 12 \\
\hline & 10 & 10 & 63 & 1 & & 10 & 95 & 6 & 16 \\
\hline & 11 & 11 & 62 & 1 & & 11 & 92 & 4 & 16 \\
\hline & 12 & 12 & 27 & 1 & & 12 & 40 & 2 & 13 \\
\hline \multirow{12}{*}{2006} & 1 & 1 & 23 & 1 & \multirow{12}{*}{2009} & 1 & 30 & 2 & 13 \\
\hline & 2 & 2 & 37 & 1 & & 2 & 52 & 5 & 15 \\
\hline & 3 & 3 & 56 & 1 & & 3 & 94 & 6 & 18 \\
\hline & 4 & 4 & 61 & 1 & & 4 & 88 & 5 & 16 \\
\hline & 5 & 5 & 80 & 1 & & 5 & 97 & 5 & 18 \\
\hline & 6 & 6 & 62 & 1 & & 6 & 81 & 5 & 15 \\
\hline & 7 & 7 & 39 & 1 & & 7 & 47 & 4 & 10 \\
\hline & 8 & 8 & 70 & 1 & & 8 & 79 & 4 & 12 \\
\hline & 9 & 9 & 70 & 1 & & 9 & 97 & 7 & 14 \\
\hline & 10 & 10 & 70 & 1 & & 10 & 87 & 7 & 16 \\
\hline & 11 & 11 & 75 & 1 & & 11 & 92 & 7 & 16 \\
\hline & 12 & 12 & 34 & 1 & & 12 & 39 & 3 & 12 \\
\hline
\end{tabular}




\begin{tabular}{|c|c|c|c|c|}
\hline \multirow{2}{*}{ Año } & \multirow{2}{*}{ Mes } & \multicolumn{3}{|c|}{ Volumen de búsqueda relativo } \\
\hline & & SciELO & Redalyc & Dialnet \\
\hline \multirow{12}{*}{2010} & 1 & 27 & 3 & 14 \\
\hline & 2 & 45 & 8 & 15 \\
\hline & 3 & 83 & 7 & 17 \\
\hline & 4 & 83 & 7 & 16 \\
\hline & 5 & 93 & 8 & 15 \\
\hline & 6 & 74 & 6 & 11 \\
\hline & 7 & 40 & 4 & 9 \\
\hline & 8 & 78 & 8 & 8 \\
\hline & 9 & 83 & 10 & 13 \\
\hline & 10 & 76 & 10 & 14 \\
\hline & 11 & 82 & 11 & 14 \\
\hline & 12 & 34 & 4 & 10 \\
\hline \multirow{12}{*}{2011} & 1 & 26 & 4 & 11 \\
\hline & 2 & 48 & 10 & 13 \\
\hline & 3 & 71 & 11 & 15 \\
\hline & 4 & 75 & 9 & 12 \\
\hline & 5 & 81 & 11 & 12 \\
\hline & 6 & 66 & 8 & 10 \\
\hline & 7 & 40 & 6 & 8 \\
\hline & 8 & 65 & 10 & 8 \\
\hline & 9 & 74 & 14 & 10 \\
\hline & 10 & 68 & 13 & 12 \\
\hline & 11 & 69 & 12 & 12 \\
\hline & 12 & 34 & 5 & 9 \\
\hline \multirow{12}{*}{2012} & 1 & 27 & 6 & 8 \\
\hline & 2 & 40 & 11 & 10 \\
\hline & 3 & 66 & 13 & 12 \\
\hline & 4 & 62 & 9 & 9 \\
\hline & 5 & 65 & 12 & 11 \\
\hline & 6 & 51 & 9 & 8 \\
\hline & 7 & 31 & 6 & 6 \\
\hline & 8 & 52 & 11 & 6 \\
\hline & 9 & 59 & 14 & 8 \\
\hline & 10 & 59 & 13 & 10 \\
\hline & 11 & 55 & 11 & 11 \\
\hline & 12 & 27 & 5 & 8 \\
\hline
\end{tabular}

\begin{tabular}{|c|c|c|c|c|}
\hline \multirow{2}{*}{ Año } & \multirow{2}{*}{ Mes } & \multicolumn{3}{|c|}{ Volumen de búsqueda relativo } \\
\hline & & SciELO & Redalyc & Dialnet \\
\hline \multirow{12}{*}{2013} & 1 & 24 & 6 & 8 \\
\hline & 2 & 37 & 11 & 9 \\
\hline & 3 & 57 & 10 & 9 \\
\hline & 4 & 64 & 11 & 10 \\
\hline & 5 & 63 & 11 & 9 \\
\hline & 6 & 52 & 8 & 7 \\
\hline & 7 & 33 & 5 & 5 \\
\hline & 8 & 52 & 10 & 5 \\
\hline & 9 & 58 & 14 & 7 \\
\hline & 10 & 58 & 13 & 10 \\
\hline & 11 & 56 & 12 & 10 \\
\hline & 12 & 25 & 4 & 8 \\
\hline \multirow{12}{*}{2014} & 1 & 22 & 6 & 7 \\
\hline & 2 & 39 & 12 & 10 \\
\hline & 3 & 53 & 12 & 11 \\
\hline & 4 & 55 & 10 & 10 \\
\hline & 5 & 62 & 12 & 10 \\
\hline & 6 & 45 & 10 & 8 \\
\hline & 7 & 32 & 6 & 6 \\
\hline & 8 & 50 & 11 & 6 \\
\hline & 9 & 62 & 15 & 8 \\
\hline & 10 & 60 & 14 & 12 \\
\hline & 11 & 57 & 12 & 11 \\
\hline & 12 & 24 & 5 & 8 \\
\hline \multirow{12}{*}{2015} & 1 & 21 & 6 & 7 \\
\hline & 2 & 33 & 13 & 10 \\
\hline & 3 & 58 & 12 & 11 \\
\hline & 4 & 59 & 11 & 10 \\
\hline & 5 & 61 & 12 & 11 \\
\hline & 6 & 51 & 9 & 8 \\
\hline & 7 & 30 & 6 & 6 \\
\hline & 8 & 47 & 10 & 5 \\
\hline & 9 & 58 & 13 & 8 \\
\hline & 10 & 54 & 12 & 11 \\
\hline & 11 & 56 & 11 & 11 \\
\hline & 12 & 24 & 5 & 8 \\
\hline
\end{tabular}




\begin{tabular}{|c|c|c|c|c|}
\hline \multirow{2}{*}{ Año } & \multirow{2}{*}{ Mes } & \multicolumn{3}{|c|}{ Volumen de búsqueda relativo } \\
\hline & & SciELO & Redalyc & Dialnet \\
\hline \multirow{12}{*}{2016} & 1 & 19 & 5 & 6 \\
\hline & 2 & 33 & 10 & 8 \\
\hline & 3 & 48 & 9 & 9 \\
\hline & 4 & 53 & 10 & 10 \\
\hline & 5 & 58 & 9 & 9 \\
\hline & 6 & 44 & 8 & 8 \\
\hline & 7 & 28 & 5 & 5 \\
\hline & 8 & 42 & 10 & 5 \\
\hline & 9 & 50 & 12 & 7 \\
\hline & 10 & 48 & 11 & 9 \\
\hline & 11 & 46 & 9 & 9 \\
\hline & 12 & 23 & 4 & 7 \\
\hline \multirow{12}{*}{2017} & 1 & 20 & 6 & 7 \\
\hline & 2 & 32 & 10 & 9 \\
\hline & 3 & 51 & 10 & 9 \\
\hline & 4 & 49 & 8 & 9 \\
\hline & 5 & 53 & 10 & 9 \\
\hline & 6 & 42 & 8 & 7 \\
\hline & 7 & 26 & 5 & 5 \\
\hline & 8 & 43 & 11 & 5 \\
\hline & 9 & 49 & 13 & 7 \\
\hline & 10 & 49 & 14 & 9 \\
\hline & 11 & 49 & 12 & 9 \\
\hline & 12 & 21 & 5 & 6 \\
\hline
\end{tabular}

\begin{tabular}{|c|c|c|c|c|}
\hline \multirow{2}{*}{ Año } & \multirow{2}{*}{ Mes } & \multicolumn{3}{|c|}{ Volumen de búsqueda relativo } \\
\hline & & SciELO & Redalyc & Dialnet \\
\hline \multirow{12}{*}{2018} & 1 & 20 & 7 & 6 \\
\hline & 2 & 29 & 11 & 8 \\
\hline & 3 & 46 & 10 & 8 \\
\hline & 4 & 52 & 11 & 9 \\
\hline & 5 & 53 & 12 & 9 \\
\hline & 6 & 42 & 9 & 7 \\
\hline & 7 & 25 & 6 & 5 \\
\hline & 8 & 39 & 10 & 5 \\
\hline & 9 & 46 & 14 & 7 \\
\hline & 10 & 43 & 12 & 8 \\
\hline & 11 & 41 & 11 & 8 \\
\hline & 12 & 18 & 5 & 5 \\
\hline \multirow{12}{*}{2019} & 1 & 17 & 6 & 6 \\
\hline & 2 & 28 & 11 & 7 \\
\hline & 3 & 37 & 9 & 7 \\
\hline & 4 & 40 & 9 & 7 \\
\hline & 5 & 45 & 11 & 8 \\
\hline & 6 & 37 & 8 & 7 \\
\hline & 7 & 22 & 6 & 5 \\
\hline & 8 & 37 & 11 & 5 \\
\hline & 9 & 48 & 14 & 8 \\
\hline & 10 & 45 & 12 & 9 \\
\hline & 11 & 41 & 11 & 9 \\
\hline & 12 & 36 & 10 & 10 \\
\hline
\end{tabular}

